\title{
CD89/CD35 Expression Ratio in Salivary Neutrophil as an Early Detection Marker for Severe Early Childhood Caries
}

\author{
Muhammad Luthfi ${ }^{1}$ Aqsa Sjuhada Oki ${ }^{1}$ Retno Indrawati ${ }^{1}$ Muhaimin Rifai ${ }^{2}$ \\ Yoes Prijatna Dachlan ${ }^{3}$ Fathilah Abdul Razak ${ }^{4}$
}

1Department of Oral Biology, Faculty of Dental Medicine, Universitas Airlangga, Surabaya, Indonesia

2Department of Physiology, Cell Culture and Animal Development, Faculty of Sciences, Brawijaya University, Malang, Indonesia

${ }^{3}$ Department of Parasitology, Faculty of Medicine, Universitas Airlangga, Surabaya, Indonesia

${ }^{4}$ Department of Oral and Craniofacial Sciences, Faculty of Dentistry, University of Malaya, Kuala Lumpur, Malaysia

Eur J Dent:2020;14:386-392

\begin{abstract}
Address for correspondence Muhammad Luthfi, Department of Oral Biology, Faculty of Dental Medicine, Universitas Airlangga, Jl. Mayjend. Prof. Dr. Moestopo 47, Surabaya 60132, Indonesia (e-mail: m.luthfi@fkg.unair.ac.id).
\end{abstract}

\author{
Abstract \\ Keywords \\ - CD89 \\ - CD35 \\ - neutrophils \\ - caries \\ - biomarker
}

Objectives To analyze CD35/CD89 expression ratio on the surface of neutrophils as an early detection marker for S-ECC.

Materials and Methods Saliva was collected from 4- to 6-year-old kindergarten students. Salivary neutrophils were obtained by instructing the subjects to rinse their mouth with $1 \mathrm{~mL}$ of sterile $1.5 \% \mathrm{NaCl}$ for 30 seconds before expectorating it into a sterile glass. The expression of $\mathrm{CFSE}^{+} \mathrm{CD}_{3} 5^{+}$and $\mathrm{CFSE}^{+} \mathrm{CD} 89^{+}$was measured and analyzed using flow cytometry.

Results The expression of $\mathrm{CFSE}^{+} \mathrm{CD} 89^{+}$in the caries-free group $(2.46 \pm 0.39)$ was significantly lower than that in the S-ECC group $(3.41 \pm 1.11)$, with a $p$-value of 0.0001 , while the expression of $\mathrm{CFSE}^{+} \mathrm{CD} 35^{+}$in the caries-free group was $(2.35 \pm 0.56)$ compared with $(1.54 \pm 0.35)(p=0.0001)$ in the S-ECC group.

Conclusions The expression ratio of $\mathrm{CFSE}^{+} \mathrm{CD} 89^{+}$and $\mathrm{CFSE}^{+} \mathrm{CD} 35^{+}$constitutes a marker for S-ECC.

\section{Introduction}

Early childhood caries (ECC) is a major problem in oral health, which affects the health of preschoolers around the world. Prevalence reaches $85 \%$ in low socioeconomic children. ECC is characterized by the presence of one or more decayed, missing, or filled primary teeth in children aged 5 years or younger. ${ }^{1}$ In children younger than 3 years, if there is caries on a smooth surface, it is an indication of severe ECC (S-ECC). From ages 3 to 5 years, one or more cavitated, missing teeth (due to caries), or filled smooth surfaces in primary maxillary anterior teeth, or decayed, missing, or filled score of $\geq 4$ (age 3 ), $\geq 5$ (age 4 ), or $\geq 6$ (age 5 ) surfaces constitutes S-ECC. ${ }^{2}$ The prevalence of ECC and S-ECC among preschool children aged 3 to 5 years in
Xinjiang reached levels of 78.2 and $41.2 \%$, respectively, which was relatively higher than the figure of $53.6 \%$ in other districts within China during the period 2010 to 2013. ${ }^{3}$ Another study by Peltzer and Mongkolchati reported the prevalence of ECC in northern Thailand in 2015 was also high as evidenced by a decayed-missing-filled teeth index score of 5.61 for ECC and 8.17 for S-ECC among preschoolers. ${ }^{4}$

In addition to affecting quality of life, ECC increases the risk of caries in permanent dentition and other oral diseases. ${ }^{5}$ In addition, performing dental treatment to the children is considerably difficult, since for children, the dentist is a nightmare. The fear of dentist, odontophobia, usually leads to postpone treatment, clinging to pharmacological therapies which produce a serious worsening of the caries itself. ${ }^{6}$ 
S-ECC, the severe form of ECC, may have a greater impact than caries during adulthood if left untreated. ${ }^{7}$ However, several cases have indicated that ECC, even S-ECC, can be prevented or regulated, given the application of appropriate measures. ${ }^{8}$

Social and behavioral risk factors which have been identified as correlated to S-ECC include: low socioeconomic status, the availability of fluoridated water, the profile of the mother (level of education, own experience of caries, and degree of awareness of dental and oral health), dietary habits, inappropriate dental health behavior, and lack of access to dental health facilities. ${ }^{9}$ These data confirmed that S-ECC predominantly affects the children of socially disadvantaged families, ethnic minorities, and individuals who live in less developed regions. ${ }^{10}$

Caries may also cause damage to the adjacent tissue due to the spread of Streptococcus mutans leading to gingival or periodontal inflammation. ${ }^{11}$ Necrotic teeth, if left untreated, may cause abscesses that spread to the adjacent tissue leading to various conditions ranging from gingivitis to cellulitis in the mandible. In the worst case scenario, the bacteria transported through the bloodstream may cause systemic infection necessitating a more expensive treatment involving type IV antibiotics. ${ }^{12}$

Recent studies have revealed a new role for neutrophils as the key component of first-line defense against microbes. ${ }^{13}$ In addition to destroying microbes through phagocytosis and releasing reactive oxygen species (ROS) and antimicrobial peptides, they also regulate immune response activation. ${ }^{14}$ Neutrophils have also been confirmed as the main contributors to proinflammatory cytokine synthesis in infected areas and chemokine and growth factors. ${ }^{15}$ Another study proved that neutrophils can initiate two-way complex interaction between macrophage and dendritic cells (DCs), natural killer cells and lymphocyte in the infected area, which further affect the innate and adaptive immune response. ${ }^{16}$

Neutrophils kill pathogenic microbes by a process of phagocytosis which is significantly more effective due to opsonization involving antibodies present on the surfaces of the microbes. Microbial phagocytosis may lead to oxidative burst, producing ROS accompanied by cytoplasmic degranulation in microbe-contained phagosome, with antimicrobial peptides and protease. ${ }^{15}$ Neutrophils express several specific receptors for fragment crystallizable $(\mathrm{Fc})$ region antibodies, such as Fc $\alpha R /$ IgA receptor (cluster of differentiation [CD]89) and complement receptor (CR)1 (CD35) that play a role in microbe recognition following opsonization. ${ }^{17}$

Complement activation leads to $\mathrm{C} 3 \mathrm{~b}$ deposition on microbe surfaces. CRs expressed on the surface of neutrophils efficiently recognize microbes bound to a complement component called CR1 (CD35). ${ }^{16}$ The abnormal phagocytosis function might be caused by several clinical disruptions, both innate and adaptive, possibly initiated by the neutrophils themselves, or complementing anomaly during opsonization. The release of premature neutrophils from the bone marrow may induce disrupted phagocytosis, possibly correlated to disruption of the neutrophils, either in terms of their quantity or function. This process may also be associated with mild to severe microbial infection, ${ }^{18}$ the risk of which may be increased by insufficient levels of neutrophil. Diseases related to the low neutrophil levels in the bloodstream include: cyclic neutropenia, chronic benign neutropenia, several congenital neutropenia, and Felty's syndrome, all of which correlate to dental and oral problems, including early tooth loss. ${ }^{19}$

Several approaches to caries prevention have been attempted including access to dental health education (specifically, the appropriate method of brushing the teeth), the provision of topical fluoride, and vaccination. ${ }^{20}$ However, to date, none of these has produced the desired outcome. This study aims to identify the risk factor of dental caries and the innate immunity mechanism which can protect against or prevent these. ${ }^{12}$

\section{Materials and Methods}

\section{Ethical Approval}

This research received the approval of the Institutional Ethical Committee (certificate no. 01/KKEPK/VII/2013), while a signed consent form was obtained from the parents of the subjects prior to the collection of data.

\section{Design and Research Procedures}

This research is an observational analytic study with cross-sectional design using S-ECC and caries free as the object of research. The sample ( 40 children) was obtained from several kindergartens in the Surabaya area. The examination of dental caries was conducted in each selected institution by measuring the decay-exfoliation-filling (def-t) index. The subjects who had been examined were subsequently divided into two groups: a caries-free group and a caries group with a def-t more than 6 . The age of all subjects ranged from 4 to 6 years at the time of the examination. Prior to specimen collection, questionnaires about the children's health had been distributed and the parents of the subjects signed a written informed consent sheet. Samples were obtained by researchers and trained personnel using a standard protocol. Subjects were not permitted to eat, drink, chew gum, or brush their teeth for 60 minutes before sampling was conducted. The samples collected were frozen (Frozen; Quanzhou, China) at a temperature of $-80^{\circ} \mathrm{C}$ for further analysis.

\section{Phagocytosis Simulation}

Observation of the phagocytosis activity of S. mutans in neutrophils was conducted by means of culturing S. mutans in a well-containing neutrophils. The simulation of phagocytosis was performed by incubating the cell suspension and bacteria in an incubator at $37^{\circ} \mathrm{C}$ and $5 \% \mathrm{CO}_{2}$ for 60 minutes. ${ }^{19}$ The cells were harvested, inserted into the microtube, and centrifuged at 2,500 rpm for 5 minutes at $4^{\circ} \mathrm{C}$. The resulting pellet was then administered with BioLegend antihuman- $\alpha$ CD89 PE, biolegend antihuman- $\alpha$ CD35 PE and biolegend antihuman- $\alpha$ CD11c-PeCy5 and these were conjugated before being pipetted. The suspension was subsequently placed into the flow cytometer cuvette, added to $300 \mu \mathrm{L}$ phosphate buffered saline, and inserted into a BD FACS caliber nozzle for 
running. The phagocytosed bacteria expressed $\mathrm{CFSE}^{+} \mathrm{CD} 89^{+}$ and $\mathrm{CFSE}^{+} \mathrm{CD} 35^{+}$measured using a flow cytometer at a wavelength of $525 \mathrm{~nm}$ with $488 \mathrm{~nm}$ excitation.

Neutrophils were obtained from saliva by instructing the subjects to rinse their mouths with $10 \mathrm{~mL}$ of sterile $1.5 \% \mathrm{NaCl}$ for 30 seconds without swallowing. The resulting fluid was expectorated in a sterile glass (Pyrex, Singapore) with the procedure being repeated four times. The collected specimens were subsequently centrifuged at $450 \mathrm{~g}$ for 15 minutes at $4^{\circ} \mathrm{C}$. The identification of neutrophils employed the use of the human neutrophil sorting cell enrichment kit (EasySep, Sheboygan).

\section{Measurement of $\mathrm{CFSE}^{+} \mathrm{CD} 35^{+}$Expression}

During the phagocytosis process, the surface receptor on the phagocytic cells can bind to the Fc fragment from one type of immunoglobulin or may bind to the complement factor (C3b). The interaction triggers a conformational change in the cytoskeleton to support the antigen ingestion process. Pathogens are destroyed by the action of oxidative and nonoxidative mediators, that is, the interaction between the complement factor of C3b or iC3b and CR (CD35). Carboxyfluorescein $\mathrm{N}$-hydroxysuccinimide ester $\left(\mathrm{CFSE}^{+}\right)$staining on bacteria carries out the function of labeling the bacteria with fluorescein isothiocyanate in order that it can be detected with a flow cytometer (Becton Dickinson; San Jose, California, United States). Meanwhile, the provision of $\mathrm{CD}^{+} 5^{+}$antibody represents a marker which indirectly confirms the observed cell during the phagocytosis process to be a neutrophil cell. Staining with $\mathrm{CFSE}^{+} \mathrm{CD} 35^{+}$is intended to detect the ability of neutrophil cells which contain CRs $\left(\mathrm{CD}^{+} 5^{+}\right)$to perform phagocytosis on bacteria (labeled $\mathrm{CFSE}^{+}$).

\section{Measurement of $\mathrm{CFSE}^{+} \mathrm{CD} 89^{+}$Expression}

Neutrophils express Fc receptor to recognize antibodies, including Fc IgA specific, which is a transmembrane glycoprotein belonging to the immunoglobulin gene superfamily. Fc $\alpha$ R or CD89 receptor is expressed by neutrophils. The staining of $\mathrm{CFSE}^{+}$in bacteria is intended to label the bacteria with
FITC fluorescence, thereby enabling its detection by means of a flow cytometer. Meanwhile, the CD89+ antibody may act as an indirect marker of the phagocytized process in neutrophils. $\mathrm{CFSE}^{+} \mathrm{CD} 89^{+}$staining is intended to detect the ability of neutrophils with $\mathrm{Fc}$ receptor $\left(\mathrm{CD} 89^{+}\right)$to phagocytize labeled bacteria $\left(\mathrm{CFSE}^{+}\right)$.

\section{Results}

The result of flow cytometry analysis is shown in - Figs. 1 and $\mathbf{2}$. The data acquired were analyzed, tabulated, and presented in mean and standard deviation forms ( - Fig. 3 ). All the participated respondents, based on the questionnaire answer, were physically and mentally healthy. Among 40 examined children, 20 of them scored def-t index more than 6 , and the rest 20 scored less than 6 . Thus, the respondents were divided into two groups, caries-free group for those with def-t index score less than 6 , and caries group for those with def-t index score more than 6. - Table 1 shows that activated neutrophils induce phagocytosis. The observation revealed a significantly lower expression of $C D 35^{+}$ in the S-ECC group, compared with the caries-free group. Meanwhile, the expression of CD89+ in the ECC group was significantly higher than the caries-free group (-Table 2 ).

\section{Discussion}

This study revealed that half of the respondents suffered from caries with def-t index score more than 6 . These results typically correspond to the available data from developing countries, which have higher prevalence of S-ECC than the developed countries, despite the prevention measures performed. ${ }^{22}$ Considering the difficulty to perform dental treatment to children due to dentophobia, ${ }^{6}$ this research is an attempt to find a way to early detect the risk of S-ECC, so the prevention measures could be performed as soon as possible.

The contents of $\boldsymbol{-}$ Table 1 indicate that salivary neutrophils contain levels of CD35 $5^{+}$expression in the S-ECC $(1.54 \%$
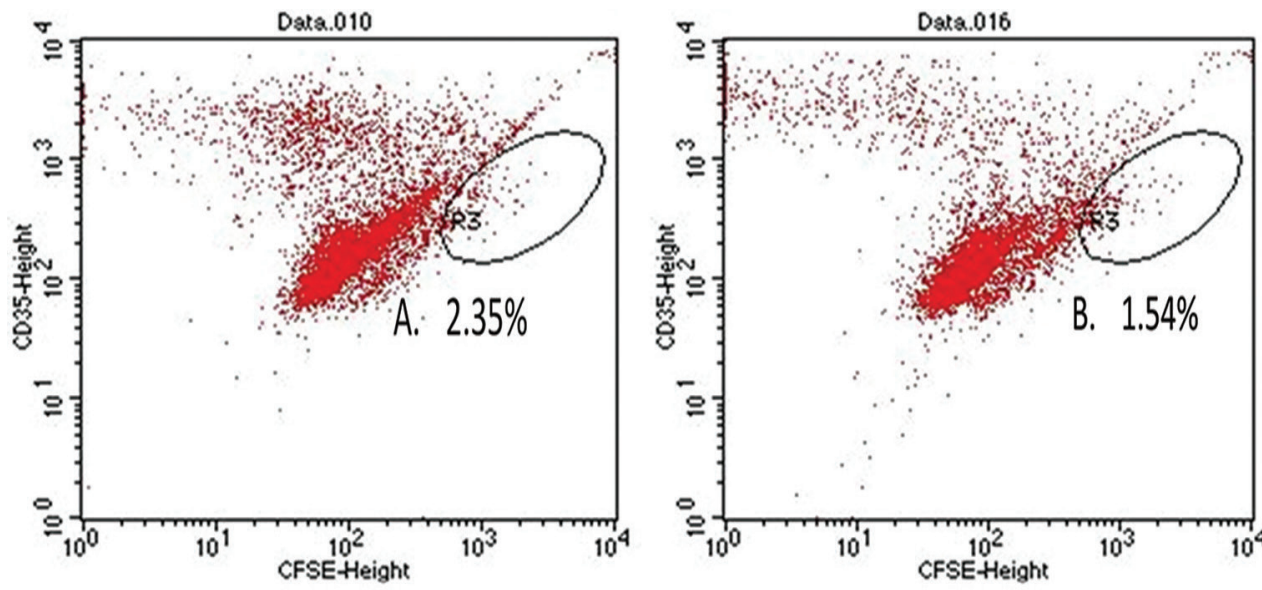

Fig. 1 Neutrophils activated to perform phagocytosis on S. mutans which were labeled with CFSE expressing CD35 ${ }^{+}$and detected using flow cytometry in early childhood free caries (A) and in early childhood caries (B). CD, cluster of differentiation. 

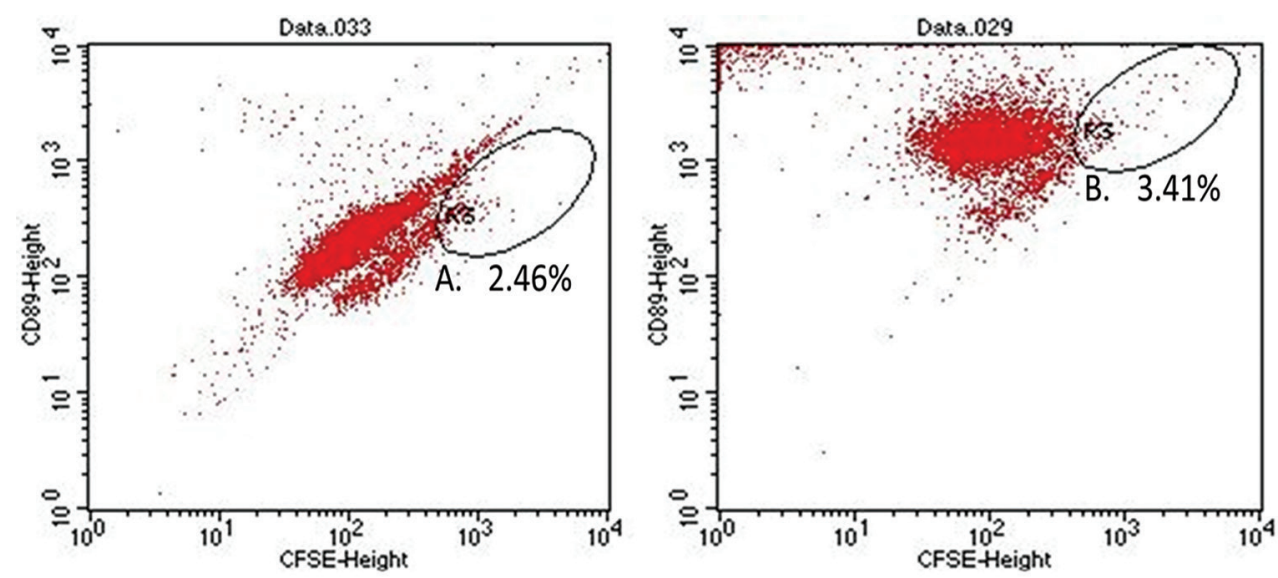

Fig. 2 The activated neutrophils phagocytizing S. mutans labeled CFSE and expressing CD89+ detected using flow cytometry in the caries-free group (A) and the S-ECC group (B). CD, cluster of differentiation; S-ECC, severe early childhood caries.

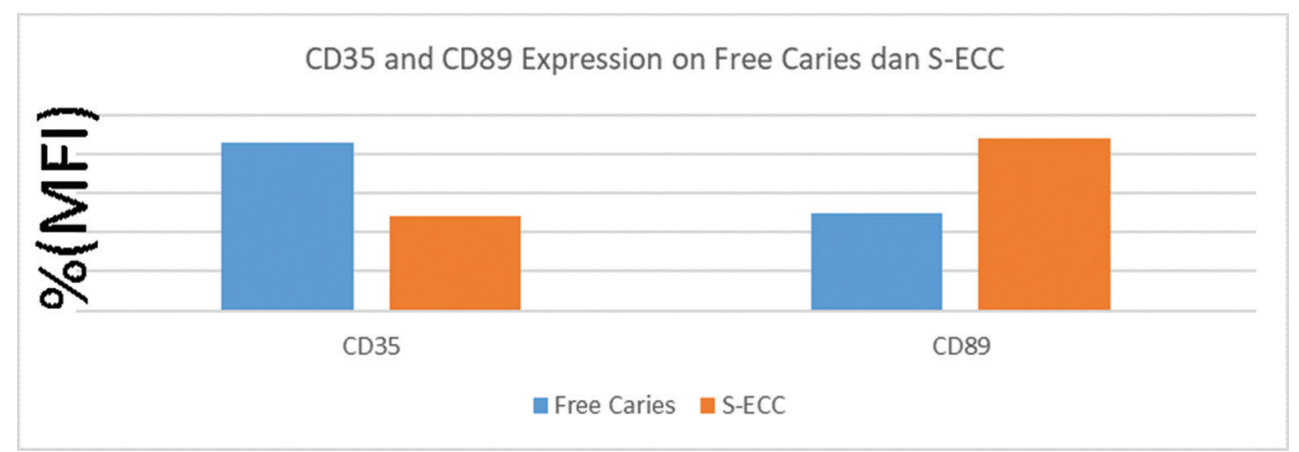

Fig. 3 Mean graph of activated neutrophils inducing phagocytosis in S. mutans labeled CFSE, expressing CD35 $5^{+}$and CD89 $9^{+}$detected using flow cytometry on the caries-free group and the S-ECC group. CD, cluster of differentiation; S-ECC, severe early childhood caries.

Table 1 Mean and standard deviation of the number of saliva neutrophils activated to perform phagocytosis of bacteria S. mutans labeled by CFSE staining and expressing $\mathrm{CD}^{+} 5^{+}$in the severe early childhood caries and caries-free (\%) teeth

\begin{tabular}{|l|l|l|l|l|}
\hline Group & N & Mean \pm standard deviation & $95 \% \mathrm{Cl}$ & $p$-Value \\
\hline Caries-free & 20 & $2.35 \pm 0.56$ & $2.09-2.61$ & $0.0001(p<\alpha)$ \\
\hline S-ECC & 20 & $1.54 \pm 0.35$ & $1.38-1.71$ & \\
\hline
\end{tabular}

Abbreviations: CD, cluster of differentiation; $\mathrm{Cl}$, confidence interval; S-ECC, severe early childhood caries.

Table 2 Mean and standard deviation of the number of saliva neutrophils activated to perform phagocytosis of bacteria S. mutans labeled by CFSE staining expressing CD89+ on the early childhood caries and caries-free (\%) teeth

\begin{tabular}{|l|l|l|l|l|}
\hline Group & $N$ & Mean \pm standard deviation & $95 \% \mathrm{Cl}$ & $p$-Value \\
\hline Free caries & 20 & $2.46 \pm 0.39$ & $2.21 \pm 2.71$ & $0.001(p<\alpha)$ \\
\cline { 1 - 2 } & 20 & $3.41 \pm 1.11$ & $2.70 \pm 4.12$ & \\
\hline
\end{tabular}

Abbreviations: $C D$, cluster of differentiation; $\mathrm{Cl}$, confidence interval; $\mathrm{ECC}$, early childhood caries.

\pm 0.35 ) group which were significantly lower than in the caries-free group $(2.35 \% \pm 0.56)$. This shows that the phagocytizing process of $S$. mutans mediated by opsonization of salivary neutrophils in S-ECC group express lower levels of $\mathrm{CD}^{+} 5^{+}$than the caries-free group. This is possibly due to the innate immunity in children with S-ECC not being able to effectively perform phagocytosis on S. mutans through complement opsonization. This lack of ability is due to the low-level deposition of C3b protein in children with S-ECC.
Therefore, the opsonization process of $S$. mutans is not optimal. This phenomenon induces a less effective phagocytosis process mediated by complement in S-ECC group compared with the caries-free group by means of observing the expression of $\mathrm{CD}^{+} 5^{+}$on the salivary neutrophil surface.

Neutrophils constitute the primary cells in the innate immune system, with anti-infection and proinflammation characteristics. In response to infection, neutrophils are the first phagocytic cells that migrate to the site of the injury to 
eliminate and microbes by means of phagocytosis, an intracellular mechanism. This process is followed by fusion of phagosome and lysosome containing antimicrobial peptide, enzyme, and reactive oxygen intermediate. Furthermore, neutrophils are also capable of destroying bacteria via extracellular mechanism by releasing antimicrobial peptide and enzymes contained in their granules. ${ }^{23}$ The surface of neutrophils express Fc IgA receptor/FcoR (CD89) which forms part of host defense against pathogens by mediating cellular response, namely, phagocytosis, oxidative burst, and release of inflammatory mediators and cytokine. ${ }^{24}$

Phagocytosis performed by neutrophils represents a complex process initiated by bacterial attachment to the neutrophil mediated by molecule opsonization, binding a component to the cell surface of bacteria and promoting phagocytosis. Protein C3b denotes crucial opsonin bound by the CR1 (CD35) in the neutrophil cell surface, causing the bacteria to penetrate the phagolysosome, inducing bacteria cell degradation. ${ }^{25}$

$\mathrm{C} 3 \mathrm{~b}$ is a molecule, produced following complement activation of the thioester group, which is highly reactive and capable of binding the microbe through the hydroxyl group or amino covalent. C3b acts as opsonin, which is subsequently recognized by $\mathrm{CD} 35$, subsequently changed into iC3b by $\mathrm{H}$ and I factors to be recognized by CR3 and CR4, also known, respectively, as Mac-1 (CD11b/CD18) and CD11c/CD18. The other possible mechanism is that of the pathogenic $S$. mutans in S-ECC evading the host immune system by forming biofilm as a physical barrier. This increases its resistance to opsonization, lysis by complement or phagocytosis. Therefore, S. mutans strain in S-ECC is more virulent compared with the caries-free group as they can modulate complement systems, the most prominent part of innate immune system which is able to recognize and opsonize $S$. mutans to be phagocytized by neutrophils.

The strategy of pathogenic bacteria to evade action of the immune system focuses mainly on the complement system which denotes the main core of innate immunity and is considered the first line of defense. The majority of pathogens in humans are known to possess a certain mechanism to evade the complement. ${ }^{25,26}$ This might also happen in S. mutans strain in individuals with S-ECC.

Based on the observations conducted, the expression of $\mathrm{CD}^{2} 9^{+}$in the S-ECC group $(3.41 \pm 1.11)$ was recorded as significantly higher compared with that in the caries-free group $(2.46 \pm 0.39)$ ( - Table 2$)$. This indicates that the phagocytizing process of $S$. mutans mediated through the opsonization process by secretory immunoglobulin A (sIgA) in the S-ECC group expressed a larger amount of CD89+ compared with the caries-free group. This is possibly due to the high antigen target being destroyed by the immune system of the S-ECC group mediated by the component of adaptive immunity as the innate system is unable to eliminate the cariogenic S. mutans bacteria.

The inability of the innate immune system in an individual with S-ECC causes the salivary neutrophils to induce a two-way interaction with the macrophage cells, DCs, natural killer cells, lymphocyte cells, and mesenchymal stem cells mediated by the complement system. ${ }^{27}$ This initiates antibody formation since neutrophils also contribute to delayedtype hypersensitivity by releasing monocyte chemotactic protein-1/CCL2 which induces the transition from an innate to adaptive immune response. ${ }^{28}$ Neutrophils constitute the component of innate immunity that induces the maturation of DC and increases the expression of costimulating molecules (HLA-DR, CD86, CD46) to produce signal inducing T-cells. The interaction of neutrophils with DC induces the production of interleukin (IL)-12 by DC and promotes the maturation and activation of T cells ( - Table 3 )..$^{29}$

SIgA represents the main mediator of the humoral immune system in the mucosal surface that is able to bind antigen and prevent infection. Meanwhile, the effector mediated by IgA is dependent on Fc $\alpha R$ (CD89) on the neutrophil surface, eliminating IgA with immune complex. ${ }^{30}$

The binding of antigen and sIgA complex to CD89 causes various immune responses, including antibody-dependent cell-mediated cytotoxicity, phagocytosis, cytokine release, oxidative burst, and degranulation. Those responses play an important role in host defense against microbes, possibly appearing as inflammation and a pathological condition. ${ }^{30}$

The phagocytosis process involving salivary neutrophils mediated by sIgA indicated by the expression of $\mathrm{CD}^{+} 9^{+}$ showed a significant increase compared with the caries-free group. However, this did not signify a cutoff in the development of caries. The increase of S. mutans in the S-ECC group with a more varying virulent strain proved capable of evading the host immune system, both innate and adaptive, despite the complex host innate immune system in saliva. ${ }^{31}$

S-ECC denotes chronic infection, producing the high level of proinflammatory cytokine C5a, IL-8, tumor necrosis factor- $\alpha$, and the formation of antigen-IgA complex due to an elevated level of antigen. Individuals with S-ECC tend to present high levels of $S$. mutans in the oral cavity, ${ }^{29}$ inducing an immune response which increases the production of immunoglobulin. This may explain the inability to prevent caries, despite the high level of SIgA. ${ }^{32}$

Table 3 The multiple regression analysis of salivary neutrophils expressing $\mathrm{CFSE}^{+} \mathrm{CD} 35^{+}$and $\mathrm{CFSE}^{+} \mathrm{CD} 89$

\begin{tabular}{|l|l|l|l|l|}
\hline Independent variable & Dependent variable & & $R^{2}$ & $p$-Value \\
\hline Neutrophil & CFSE-CD35 & Caries free & 0.785 & $<0.0001$ \\
\cline { 3 - 5 } & & S-ECC & 0.539 & $<0.0001$ \\
\hline Neutrophil & CFSE-CD89 & Caries free & 0.771 & $<0.0001$ \\
\cline { 3 - 5 } & & S-ECC & 0.753 & $<0.0001$ \\
\hline
\end{tabular}

Abbreviation: S-ECC, severe early childhood caries. 
The destructive function of the antibody Fc region will not occur if the pathogenic bacteria bind to the Fc region. Group A streptococci contain fibronectin-binding protein I (SfblI) that is capable of binding to the Fc region of IgG, preventing phagocytosis. ${ }^{33}$ Several forms of M protein also demonstrate the ability to bind the Fc domain of IgG and IgA. ${ }^{34} \mathrm{~A}$ further study is required to establish whether $S$. mutans possesses such ability, considering that the high expression of CD89 in salivary neutrophils does not reflect the efficacy of phagocytizing process mediated by sIgA opsonization. It can be concluded from this study that the high level of S. mutans in individuals with S-ECC facilitates the uninterrupted progress of caries.

The limitation of this study is we did not perform a detailed observation of the children feeding habit, infant feeding practices, nutritional intake, and the parents' oral hygiene practice. These factors, of course, may affect the prevalence of caries and how the host immune responds. Thus, future research is necessary to find whether this early detection marker is affected by aforementioned factors.

\section{Conclusions}

The ratio of $\mathrm{CD} 89^{+} / \mathrm{CD} 35^{+}$expression constitutes a marker early detection of S-ECC.

\section{Note}

All the listed authors have read the manuscript and hereby stated that this manuscript has not been presented in any conference/convention/meeting.

\section{Funding}

This research was funded by the Directorate of Research and Community Services of Directorate General of Research and Development Strengthening from Ministry of Research, Technology and Higher Education of the Republic of Indonesia.

\section{Conflict of Interest}

None declared.

\section{Acknowledgment}

The authors would like to thank Prof. Muhaimin Rifa'i, $\mathrm{PhD}$, MedSc, for his invaluable support in conducting this research.

\section{References}

1 Feldens CA, Giugliani ER, Duncan BB, Drachler MdeL, Vítolo MR. Long-term effectiveness of a nutritional program in reducing early childhood caries: a randomized trial. Community Dent Oral Epidemiol 2010;38(4):324-332

2 Suzuki N, Yoneda M, Naito T, Iwamoto T, Hirofuji T. Relationship between halitosis and psychologic status. Oral Surg Oral Med Oral Pathol Oral Radiol Endod 2008;106(4):542-547

3 Zhang X, Yang S, Liao Z, et al. Prevalence and care index of early childhood caries in mainland China: evidence from epidemiological surveys during 1987-2013. Sci Rep 2016;6:18897

4 Peltzer K, Mongkolchati A. Severe early childhood caries and social determinants in three-year-old children from
Northern Thailand: a birth cohort study. BMC Oral Health 2015;15(1):108

5 Masumo R, Bardsen A, Mashoto K, Åstrøm AN. Prevalence and socio-behavioral influence of early childhood caries, ECC, and feeding habits among 6-36 months old children in Uganda and Tanzania. BMC Oral Health 2012;12(1):24

6 De Stefano R. Psychological factors in dental patient care: odontophobia. Medicina (Kaunas) 2019;55(10):678

7 Sachdev J, Bansal K, Chopra R. Effect of comprehensive dental rehabilitation on growth parameters in pediatric patients with severe early childhood caries. Int J Clin Pediatr Dent 2016;9(1):15-20

8 Doğan D, Dülgergil CT, Mutluay AT, Yıldırım I, Hamidi MM, Çolak H. Prevalence of caries among preschool-aged children in a central Anatolian population. J Nat Sci Biol Med 2013;4(2):325-329

9 Bramantoro T, Karimah N, Sosiawan A, et al. Miswak users' behavior model based on the theory of planned behavior in the country with the largest Muslim population. Clin Cosmet Investig Dent 2018;10:141-148

10 Custodio-Lumsden CL, Wolf RL, Contento IR, et al. Validation of an early childhood caries risk assessment tool in a low-income Hispanic population. J Public Health Dent 2016;76(2):136-142

11 Califano JV; Research, Science and Therapy Committee American Academy of Periodontology. Position paper: periodontal diseases of children and adolescents. J Periodontol 2003;74(11):1696-1704

12 Luthfi M, Setijanto D, Rahardjo MB, et al. Correlation between human neutrophil peptide 1-3 secretion and azurophilic granule (CD63) expression in early childhood caries. Dent Res J (Isfahan) 2019;16(2):81-86

13 Elbim C, Katsikis PD, Estaquier J. Neutrophil apoptosis during viral infections. Open Virol J 2009;3(1):52-59

14 Mortaz E, Alipoor SD, Adcock IM, Mumby S, Koenderman L. Update on neutrophil function in severe inflammation. Front Immunol 2018;9:2171

15 Jaillon S, Galdiero MR, Del Prete D, Cassatella MA, Garlanda C, Mantovani A. Neutrophils in innate and adaptive immunity. Semin Immunopathol 2013;35(4):377-394

16 Kobayashi SD, Voyich JM, Burlak C, DeLeo FR. Neutrophils in the innate immune response. Arch Immunol Ther Exp (Warsz) 2005;53(6):505-517

17 Albrechtsen M, Yeaman GR, Kerr MA. Characterization of the IgA receptor from human polymorphonuclear leucocytes. Immunology 1988;64(2):201-205

18 Nobile CG, Fortunato L, Bianco A, Pileggi C, Pavia M. Pattern and severity of early childhood caries in Southern Italy: a preschool-based cross-sectional study. BMC Public Health 2014;14(1):206

19 Mayadas TN, Cullere X, Lowell CA. The multifaceted functions of neutrophils. Annu Rev Pathol 2014;9:181-218

20 Yunanto A, Endharti AT, Widodo A. Neutrophil, TLR2, and TLR4 expression in newborns at risk of sepsis. Paediatr Indonesia 2013;53(3):132

21 Gasparoto TH, Vieira NA, Porto VC, Campanelli AP, Lara VS. Differences between salivary and blood neutrophils from elderly and young denture wearers. J Oral Rehabil 2011;38(1):41-51

22 Astuti ESY, Sukrama IDM, Mahendra A. Innate immunity signatures of early childhood caries (Ecc) and severe early childhood caries (S-Ecc) Biomed Pharmacol J 2019;12(3):1129-1134

23 Borregaard N. Neutrophils, from marrow to microbes. Immunity 2010;33(5):657-670

24 Monteiro RC, Van De Winkel JG. IgA Fc receptors. Annu Rev Immunol 2003;21(1):177-204

25 Sarantis H, Grinstein S. Subversion of phagocytosis for pathogen survival. Cell Host Microbe 2012;12(4):419-431 
26 Potempa M, Potempa J. Protease-dependent mechanisms of complement evasion by bacterial pathogens. Biol Chem 2012;393(9):873-888

27 Plitas G, Rudensky AY. Regulatory T cells: differentiation and function. Cancer Immunol Res 2016;4(9):721-725

28 Yang P, Li Y, Xie Y, Liu Y. Different faces for different places: heterogeneity of neutrophil phenotype and function. J Immunol Res 2019;2019:8016254

29 Schuster S, Hurrell B, Tacchini-Cottier F. Crosstalk between neutrophils and dendritic cells: a context-dependent process. J Leukoc Biol 2013;94(4):671-675

30 Woof JM, Russell MW. Structure and function relationships in IgA. Mucosal Immunol 2011;4(6):590-597

31 Luthfi M, Indrawati R, Arundina I, Dachlan YP. Korelasi Jumlah Streptococcus mutans(S. mutans) dan Level Ekspresi Interlukin
8 (IL-8) pada Severe Early Childhood Caries. Maj Kedokt Gigi Indones 2015;20(2):142

32 Al Amoudi N, Al Shukairy H, Hanno A. A comparative study of the secretory IgA immunoglobulins (s.IgA) in mothers and children with SECC versus a caries free group children and their mothers. J Clin Pediatr Dent 2007;32(1):53-56

33 Miyoshi-Akiyama T, Takamatsu D, Koyanagi M, Zhao J, Imanishi K, Uchiyama T. Cytocidal effect of Streptococcus pyogenes on mouse neutrophils in vivo and the critical role of streptolysin S. J Infect Dis 2005;192(1):107-116

34 Brenot A, King KY, Janowiak B, Griffith O, Caparon MG. Contribution of glutathione peroxidase to the virulence of Streptococcus pyogenes. Infect Immun 2004;72(1):408-413 